\title{
I Solenni Funerali di Filippo V nella cattedrale di Palermo
}

\author{
Emanuela Garofalo
}

\section{SINTESI}

Fra il mese di settembre del 1746 e il mese di gennaio del 1747, furono celebrate nella cattedrale di Palermo, per l'estinto monarca Filippo V di

Borbone, due distinte cerimonie funebri, espressione di altrettante sfere di potere, rispettivamente quella religiosa e quella civile. II primo dei due funerali fu più modesto a causa dell'esiguità dei fondi a disposizione dei committenti. L'incarico per la realizzazione del catafalco e dell'apparato effimero, con cui si andarono a ricoprire i sostegni della navata centrale, stranamente non $f u$

affidato a un architetto ma a un pittore, Gaspare Fumagalli. II risultato complessivo dell'opera di quest'ultimo, nonostante l'esistenza di un'incisione che ritrae il catafalco, in parte sfugge, essendo troppo generica la descrizione in nostro possesso relativa all'apparato per la navata principale.

Ben più sontuosa fu la cerimonia funebre realizzata, interamente a spese del Real Patrimonio, nel gennaio del 1747. L'allestimento interno della cattedrale e il catafalco collocato nella stessa, entrambi ritratti

\section{ABSTRACT}

Two separate funerals were celebrated in Palermo's Cathedral for the death of King Philip the $V$ of Borbone between September 1746 and January 1747. The two ceremonies were the expression of the two major authorities, the Church and the Government. The first funeral was more humble because of the narrow funds of the purchasers. The appointment to build the catafalque and the ephemeral apparatus that covered the supports of the central nave was unusually not committed to an architect but to a painter, Gaspare Fumagalli. We are partly not aware of the final result of his work, in spite of the existence of an engraving which portrays the catafalque, because the description we have about the main nave is too generic.

The funeral held in January 1747 , entirely at the expense of the Royal Treasury, was much more sumptuous. In this case the interior fitting of the Cathedral and the catafalque are both represented by 
da accurate incisioni sono, questa volta, opera di un noto architetto, Nicoló Palma, cui spettano nell'anno in questione i titoli di Architetto del

Senato e Ingegnere del Real Patrimonio. L'analisi del complesso delle architetture effimere realizzate dal Palma in questa occasione ha consentito di avviare diversi ragionamenti, non soltanto di carattere stilistico e linguistico, ma che coinvolgono anche l'edificio della cattedrale di Palermo, e in particolare

la sua spazialità interna. L'apparato realizzato dal Palma per la navata centrale della stessa, infatti, potrebbe celare una prima proposta progettuale per la trasformazione di uno spazio che, per la sua eccessiva eterogeneità, doveva già risultare inaccettabile.

Lo studio dei due funerali in esame ha preso le mosse da un'attenta rilettura dei testi a stampa, corredati da incisioni, prodotti in tali occasioni. Essi, oltre alle dettagliate descrizioni delle cerimonie e degli apparati effimeri prodotti per le stesse, contengono una serie di informazioni che offrono stimolanti occasioni di riflessione anche su temi di più ampio respiro. accurate carvings and they were made by a well-known Architect of the Senate and Engineer of the Royal Treasury. The analysis of the structures made by Palma in this occasion has given the opportunity of starting several remarks, not only from a stylistic and a linguistic point of view, but they also concern the building of Palermo's Cathedral and its internal spatiality. The apparatus made by Palma for its central nave, in effect, could conceal the first project proposal to transform a space that was unacceptable due to its excessive heterogeneity. The study of the above mentioned two funerals started from an attentive rereading of the printed texts, accompanied by carvings, produced in these occasions. Beyond accurate descriptions of the funerals and of the related ephemeral apparatus, they include several information giving interesting occasions to consider wider-ranging themes.

“L'avviso della morte del re cattolico Filippo $V{ }^{1}$ giunse a Palermo il primo agosto del 1746 e fu da quel momento, per i successivi sei mesi, «ordinato rigoroso lutto per la nobiltà e per le signore dame di questa città di Palermo e del regno» 2. In questo modo il marchese di Villabianca inserisce nei suoi «diari» la notizia della scomparsa dell'«Augusto Re», cui seguono, nelle successive pagine, annotazioni relative ai riti funebri che, per

Cfr. F.M. Emanuele e Gaetani marchese di Villabianca, Diario Palermitano, dal / gennaio 1746 al 31 dicembre 1758, in Diari della città di Palermo dal secolo xvi al xix, a cura di G. Di Marzo, vol. XII, Palermo 1874, pag. 42.

ibidem. 
lo stesso monarca, furono celebrati nella città di Palermo, nei mesi successivi al funesto «avviso». Dalle semplici messe di suffragio ai sontuosi funerali, numerose furono le iniziative promosse, in tal senso, dalle principali autorità cittadine ${ }^{3}$.

Un dato, trascurato da chi ha trattato l'argomento in precedenza ${ }^{4}$, emerge in modo evidente dagli scritti del Villabianca: nella cattedrale di Palermo, a distanza di pochi mesi, furono celebrati, secondo il rituale romano dei «novendiali ecclesiastici» e con la realizzazione di apparati effimeri e catafalchi, due funerali distinti, la cui memoria, oltre che dai già citati "diari» del Villabianca e dalla continuazione di quelli, più o meno coevi, del Mongitore, a opera di Francesco Serio e Mongitore ${ }^{5}$, è stata trasmessa da due testi a stampa, corredati da accurate incisioni ${ }^{6}$.

Nel giro di quattro mesi, cioè, in relazione a un unico evento funesto, la medesima chiesa cattedrale del capoluogo siciliano venne per ben due volte «apparata a lutto», con la realizzazione di distinti apparati effimeri per la nave principale e di due catafalchi, fra loro molto differenti, impegnando a tal fine ingenti capitali. Sembra, infatti, che, nonostante il breve tempo intercorso e il comune soggetto defla celebrazione, nulla di ciò che

3 Le celebrazioni di cui dà notizia il Villabianca sono, nell'ordine: un funerale celebrato nel duomo il 19 agosto del 1746, ordinato dall'arcivescovo di Palermo; una messa di requiem celebrata il 27 agosto del 1746 nella piccola chiesa dell'Albergo dei poveri, con l'intervento dei deputat; il primo solenne funerale nella Cattedrale, tutta «apparata a lutto», dal 16 settembre del 1746; un funerale solenne, anche in questo caso con chiesa "apparata a lutto", celebrato dal "capitolo della real cappella di S. Pietro di Palazzo", nella cappella stessa, il 13 gennaio del 1747, e, infine, il secondo e più sontuoso solenne funerale celebrato in Cattedrale, su ordine regio, a spese del Tribunale del Real Patrimonio, a partire dal 16 gennaio del 1747. Cfr. F.M. EMANUELE E GAETAN। MARCHESE DI VILLABIANCA, Diario..., cit., pagg. 42-66.

4 Fra i testi che riportano notizie utili sulle celebrazioni funebri in onore di Filippo $V$ effettuate a Palermo, oltre ai già citati «Diari Palermitani», segnaliamo in particolare: G.E. DI BLASi E Gambaconta, Storia cronologica de' Viceré, luogotenenti, e presidenti del Regno di Sicilia, Palermo 1791; F. M. EMANUEle E GAeTANI MARCHESE DI VILLABIANCA, Le feste reali di Sicilia nel secolo Xvili, ms. del XvIli sec., pubblicato a cura di M.C. Ruggien! Tricoli, Palermo 1991; G. Isgro, Feste barocche a Palermo, Palermo 1986; M.C. RuGGIERI TRlCOLI, II "funeral teatro". Apparati e mausolei effimeri dal xvil al $x \times$ secolo a Palermo, Palermo 1993.

5 A. Mongitore, Diario Palermitano dal 6 gennaio del 1737 al 19 maggio del 1743 , con la continuazione fino agli 11 di novembre del 1751 di Francesco Serio e Mongitore, in Diari della ..., cit., a cura di G. Di MARzo, vol. X, Palermo 1872, pagg. 122-125.

6 I testi cui si fa riferimento, entrambi anonimi (anche se per il secondo il Villabianca indica, nel suo manoscritto Le feste reali di Sicilia nel secolo xvill, pubblicato, a cura di M.C. RugGIERI TRICOLI, a Palermo nel 1991, come autore Padre Francesco Tamburini), sono rispettivamente: Relazione breve delle solenni esequie di Filippo V, re delle Spagne, celebrate dal Capitolo e dal Clero della Metropolitana chiesa di Palermo, Palermo 1746; Esequie Reali per la morte dell'Augusto Re Cattolico Filippo V Borbone solennemente celebrate nella Metropolitana chiesa di Palermo Capitale del Regno per comandamento della Sacra Real Maestà di Carlo Infante delle Spagne Re delle due Sicilie, di Gerusalemme, Duca di Parma, Piacenza, e Castro, Gran Principe ereditario di Toscana..., Palermo 1747. 


\section{ESEQUIE REALI}

DELL AUGUSTO RE CATTOLICO

DELLE SPAGNE

\section{FILIPPO QUINTO \\ $\mathrm{B} O \mathrm{R} B O \mathrm{NE}$}

SOLE TNEAE

Neha Letrophirana Chiefa di Palermo Capitale del Regno

PER COMARDAMENTO

TILIA SACRA REAL MAESTA

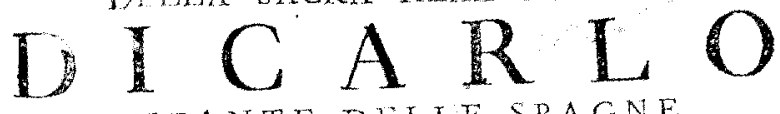

INFANTE DELLE SPAGNE

Re delle due Siciic, di Gerufuemme, Duca di Parma, Pacenza, c Calto, cran Principe exeditario di Tolcana $8 \mathrm{c}$.

FIGL DELLO STESSO DEFUNTO WONARCA

A fpete del Real Patrimonio

P.R ORDINE

DEL REGIO MEDESIMO TRIBUNALE.

DESCRITTEE DEDICATE

ALIA STESSA S. R. M. S.

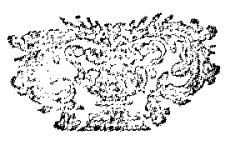

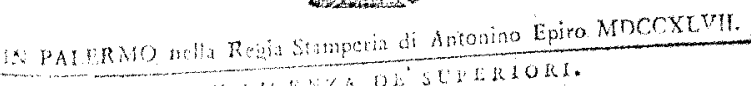

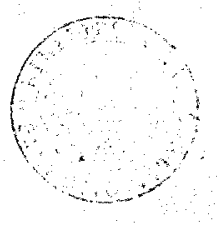


era stato approntato per il primo funerale venne riutilizzato nella seconda e più solenne funzione ${ }^{7}$.

Per questa procedura apparentemente insolita è, in realtà, riscontrabile un antecedente nei funerali di Carlo $1{ }^{8}$, ai quali, per altro, si fa più volte riferimento nel testo del $1747^{\circ}$. La spiegazione più logica è legata alla differente committenza delle due celebrazioni e, in generale, a ragioni politiche, essendo, quella dei funerali regi, un'occasione di autocelebrazione per i soggetti promotori, ma anche un pretesto per rendere omaggio, oltre che all'estinto, al monarca vigente.

Il primo dei due funerali fu celebrato "dal Capitolo e dal Clero della Metropolitana Chiesa di Palermo" ${ }^{10}$, particolarmente riconoscente a Filippo $V$ per aver concesso agli stessi «in accrescimento di loro quotidiane distribuzioni due annue pensioni» ${ }^{11}$, rispettivamente "di scudi 2000 sopra l'arcivescovado di Palermo; e l'altra di 1000 sopra quello di Monreale» ${ }^{12}$. E' chiaro quindi che le principali autorità ecclesiastiche cittadine vollero farsi promotrici, in prima persona in modo autonomo, di una solenne celebrazione in memoria del defunto monarca, impegnando a tal fine il più rappresentativo edificio ecclesiastico della città, la sua «chiesa Metropolitana». E' probabile che, al di là di una reale espressione di gratitudine nei confronti di Filippo $\mathrm{V}$, il funerale del 1746 fosse, sostanzialmente, una mossa politica per ottenere dal regnante sovrano, Carlo di Borbone, uguali agevolazioni economiche, rammentandogli e sottoponendo alla pubblica attenzione la questione delle «pensioni» sopra menzionate delle quali, fra l'altro, nella Relazione prodotta in tale occasione, si dice: "La prima...ebbe tosto effetto mercè l'amore, onde fu sempre portato a favor della sua Cattedrale l'arcivescovo Giuseppe Gasch di sempre gloriosa e venerabile memoria: e l'altra resta tuttavia di essere assegnata" ${ }^{13}$.

\footnotetext{
Especificato, nei documenti relativi alle gare d'appalto per la realizzazione degli apparati effimeri per il funerale del 1747, che i materiali da utilizzare per gil stessi devono essere «nuovi»: cfr. M.C. Ruggieri Tricol, // «funeral..., cit., Palermo 1993, pag. 26.

8 Ivi, pagg. 81 e 82; «Dopo alcune manifestazioni di cordoglio delle quali resta traccia attraverso un disegno di catafalco firmato da Giacomo Amato per il 7 novembre 1700, nell'aprile dell'anno successivo vengono celebrate a Palermo le esequie di Carlo II con "magnificenza straordinaria e pompa veramente regia», fastosità che si manifesta nell'apparato funebre della cattedrale...».

9 Esequie Reali per la morte dell'Augusto Re Cattolico Filippo V..., cit.; i riferimenti alle esequie di Carlo II sono presenti in più parti del testo, fra le quali si segnala in particolare la nota inserita fra le pagg. 39 e 40 .

${ }_{10}$ Relazione breve delle solenni esequie di Filippo $V$..., cit., pag. 1.

11 Ibidem.

12 Ibidem

13 lbidem.
} 
II secondo funerale, invece, fu commissionato dallo stesso monarca, Carlo di Borbone, con dispaccio del 17 settembre del 1746, all'indomani cioè del primo funerale ${ }^{14}$, ordinando "che si celebrassero reali, e pompose esequie...nella Capitale, nella stessa forma, come si erano fatte nella morte di Carlo $\mid \|_{\gg{ }^{15}}$. L'ordine fu a sua volta trasmesso dal viceré principe Corsini al Tribunale del Real Patrimonio il giorno 27 dello stesso mese ${ }^{16}$. Gli esponenti di tale Tribunale si attivarono per eseguire la volontà del re «addossando l'affare... al Signor D. Giovanni Maria Ramondetta Duca di Montalbo...Uomo Patrizio noto, e sperimentato in altre congiunture d'importanza» ${ }^{17}$. In questo secondo caso si trattò, quindi, di un vero e proprio "funerale di stato", alla cui realizzazione concorsero le principali autorità e istituzioni amministrative cittadine, nonché le più alte cariche dello stato, nell'intento di soddisfare la volontà e le aspettative del re. La celebrazione di due distinti funerali si spiega pertanto come espressione delle due sfere di potere, quella ecclesiastica e quella civile, entrambe impegnate a mostrare la propria solerzia e a compiacere il sovrano, con fasto e magnificenza proporzionati alle risorse finanziarie a disposizione delle stesse.

II funerale promosso dal Capitolo e dal Clero, dovendo contare esclusivamente sui fondi di pertinenza degli stessi, non poté competere, quanto a sontuosità, con quello organizzato successivamente, interamente a spese del Real Patrimonio. La celebrazione del primo fu intrapresa a partire dal 16 settembre del 1746, avendo cominciato già «...la sera precedente a sonare a mortorio tutte le campane della Cattedrale... seguitate dalla maggior parte di quelle dell'altre chiese della città; essendosi fatto l'istesso la notte e la mattina vegnente" ${ }^{18}$. La pubblicazione di un piccolo fascicolo, corredato da una incisione, a opera del tipografo Felicella, nello stesso anno in cui fu celebrato il funerale in questione, consente di ricostruire le opere effimere realizzate in tale circostanza all'interno della cattedrale. In particolare, per quel che concerne l'apparato realizzato per la navata principale, si tratta di una ricostruzione alquanto approssimativa essendo la

14 Riteniamo che non si tratti di una semplice coincidenza, ma quasi di una sorta di risposta da parte del sovrano al «Clero metropolitano», dettata da una volontà di autocelebrazione della propria persona e della propria carica, mettendo in scena un grandioso spettacolo in cui, come riferisce Padre Tamburini nella relazione dallo stesso prodotta in occasione del funerale (Esequie Reali per la morte dell'Augusto Re Cattolico Filippo V..., cit., pag. 39), «senza perdonare a magnificenza si avesse solamente davanti gli occhi una funzione, che fusse degna della memoria di un re...".

15 G.E. DI Blasi E Gambacorta, Storia cronologica..., cit., pag. 410.

16 Ibidem; l'intera vicenda è riportata, inoltre, con dovizia di particolari e comprensiva della trascrizione dell'ordine trasmesso dal viceré al Tribunale del Real Patrimonio, nella voluminosá relazione: Esequie Reali per la morte dell'Augusto Re Cattolico Filippo V..., cit., pagg. 39 e 40.

Ivi, pagg. 42-45.

18 Relazione breve delle solenni esequie di Filippo $V . .$. cit., pag. 1. 
memoria di quest'ultimo affidata esclusivamente a una descrizione di poche righe, contenuta nel fascicolo di cui sopra. Nello stesso si legge: «Si trovò nel di prefisso il Duomo tutto apparato di lutto, ma in una nuova foggia, e molto dicevole alla maestà della funzione; perché i neri panni erano tramezzati, e cinti da zoccoli, cornici, scudi sostenuti da puttini di rilievo, e capitelli tutti inargentati, che formavano, e adornavano i pilastri, gli archi, e il cornicione del magnifico Tempio: e vi erano da pertutto distribuite dell'imprese allusive all'idea della funebre pompa" ${ }^{19}$. Da queste poche righe si deduce che, oltre all'inserimento di panni neri e di vari elementi decorativi, bidimensionali e a tutto tondo, fu approntato, come era consuetudine, anche un finto rivestimento architettonico per la navata centrale. Zoccoli, cornici e capitelli andarono a ricoprire i sostegni, gli archi e il cornicione esistenti, conferendo un nuovo aspetto all'interno della chiesa. L'assenza di qualsiasi riproduzione grafica di tale apparato e di una descrizione più accurata rende purtroppo impossibile una dettagliata ricostruzione dello stesso e non consente inoltre di effettuare un confronto con quanto venne predisposto appena quattro mesi dopo, in occasione del secondo funerale celebrato in cattedrale per Filippo $\mathrm{V}$.

Diversa è la situazione per quel che concerne il catafalco al quale si riferisce l'incisione del Bova, allegata al fascicoletto pubblicato in occasione del funerale. All'interno di quest'ultimo, di seguito al passo riportato in precedenza, troviamo una sintetica descrizione del catafalco: "Sorgeva in mezzo alla gran Nave alta macchina tutta di pittura ${ }^{20}$, e divisa in tre ordini con varie figure rappresentanti le virtù che più risplendettero nel defunto sovrano. In cima di essa vedevasi il ritratto di lui che veniva coronato dalla Gloria, sotto ricca cortina sostentata da molti puttini e che arrivava fino al tetto. Ardeva intorno alla gran macchina grande, e corrispondente quantità di torce» ${ }^{21}$. Osservando l'incisione possiamo, inoltre, aggiungere che si trattava di una macchina di dimensioni imponenti, in apparenza massiccia, priva di qualsiasi forma di spazialità interna. Era composta da un elemento centrale ad andamento piramidale, costituito cioè da tre ordini sovrapposti di dimensioni decrescenti, preceduto da tre gradini mistilinei, al

19 Ibidem.

20 La frase sopra riportata indurrebbe a pensare che potrebbe essersi trattato anche di un semplice dipinto illusionistico, raffigurante una massiccia "macchina funebre», e non di un catafalco vero e proprio. Gli elementi attualmente a nostra disposizione sono, comunque, insufficienti per spingerci al di là di una semplice ipotesi, la quale, per altro, se da un canto fornisce la più convincente giustificazione per la scelta da parte dei committenti di affidare l'incarico a un pittore (come vedremo più avanti), d'altro canto risulta piuttosto fragile alla luce di una serie di altre considerazioni, legate in primo luogo alle modalità di svolgimento del solenne rito funebre.

21 Relazione breve delle solenni esequie di Filippo $V . .$. , cit., pag. 2. 
quale erano annessi quattro piedistalli a base rettangolare posti in diagonale, sormontati da altrettante statue. Alla base della «macchina» era presente il motivo decorativo del teschio e delle ossa incrociate, abbastanza frequente negli apparati funebri; per il resto era assente qualsiasi riferimento a motivi macabri. II tutto era arricchito dall'inserimento di vasi, dai quali si sprigionavano essenze profumate, e da decorazioni di varia natura, fra cui corazze, lance e scudi a tutto tondo nella parte superiore del catafalco, prima dell'ultimo corpo di coronamento. Fra gli elementi decorativi erano presenti, inoltre, uno scudo con i gigli e, in alto, l'aquila recante sul petto uno stemma coi diversi scudi dei regni dominati dal defunto monarca, nonché motivi vegetali. Mensole a voluta sostenevano la cornice aggettante posta al di sopra del primo e più massiccio corpo della «macchina». Nella zona di coronamento erano inseriti il simbolico cuscino, sul quale erano deposti corona e scettro, e più in alto, fra nuvole e angeli, il ritratto di Filippo $\mathrm{V}$, come riportato nel passo della relazione citato in precedenza. La grande quantità di torce, cui si fa riferimento nello stesso passo, era disposta tutt'intorno al basamento, nei piedistalli rettangolari e, in generale, nella parte superiore della «macchina». Nella relazione sono riportate, inoltre, le iscrizioni inserite nel catafalco, una in corrispondenza del «...secondo ordine sopra l'armi della Real Casa Borbone» ${ }^{22}$, le altre a formare un «... elogio, contenente le principali azioni del defunto principe... a quattro lati della macchina ripartito in quattro gran scudi» 23.

La relazione non fa alcun riferimento all'artefice dell'apparato e del catafalco, ma nell'incisione di Bova, in basso a destra, è indicato come inventore di quest'ultimo Gaspare Fumagalli ${ }^{24}$. Possiamo ipotizzare, pertanto, che sia stato disegnato dallo stesso anche l'apparato effimero per la navata principale della cattedrale. Si tratta di un «... pittore ornamentista e di architettura, scenografo e quadraturista e paesista» ${ }^{25}$, nato a Roma e trasferitosi a Palermo, in età giovanile, intorno agli anni trenta del Settecento. Sembrerebbe sorprendente, ed effettivamente è alquanto inconsueto, che per una celebrazione di tale importanza la realizzazione degli apparati effimeri non sia stata commissionata a un architetto ma a un

22 Ibidem.

23 Ivi, pag. 3

24 Nell'incisione si legge: «Mausoleo eretto dal R.mo Capitolo e dall'insigne Clero della Metropolitana Chiesa di Palermo per le solenni esequie di Filippo V. Gaspar Fumagalli Archit. Inv. del. Sac. Ant. Bova Sc. 1746».

25 M. GutTIlla, Fumagalli Gaspare, voce in L. Sarullo, Dizionario degli artisti siciliani. Pittura, vol.II, a cura di M.A. SPADARO, Palermo 1993, pag. 213; in proposito vedi anche: A. Gallo, Notizie intorno agli architetti siciliani ed agli esteri soggiornanti in Sicilia, ms. sec. XIX, f. 491, Biblioteca Regionale Siciliana, ai segni Qq D 42 n. 173. 


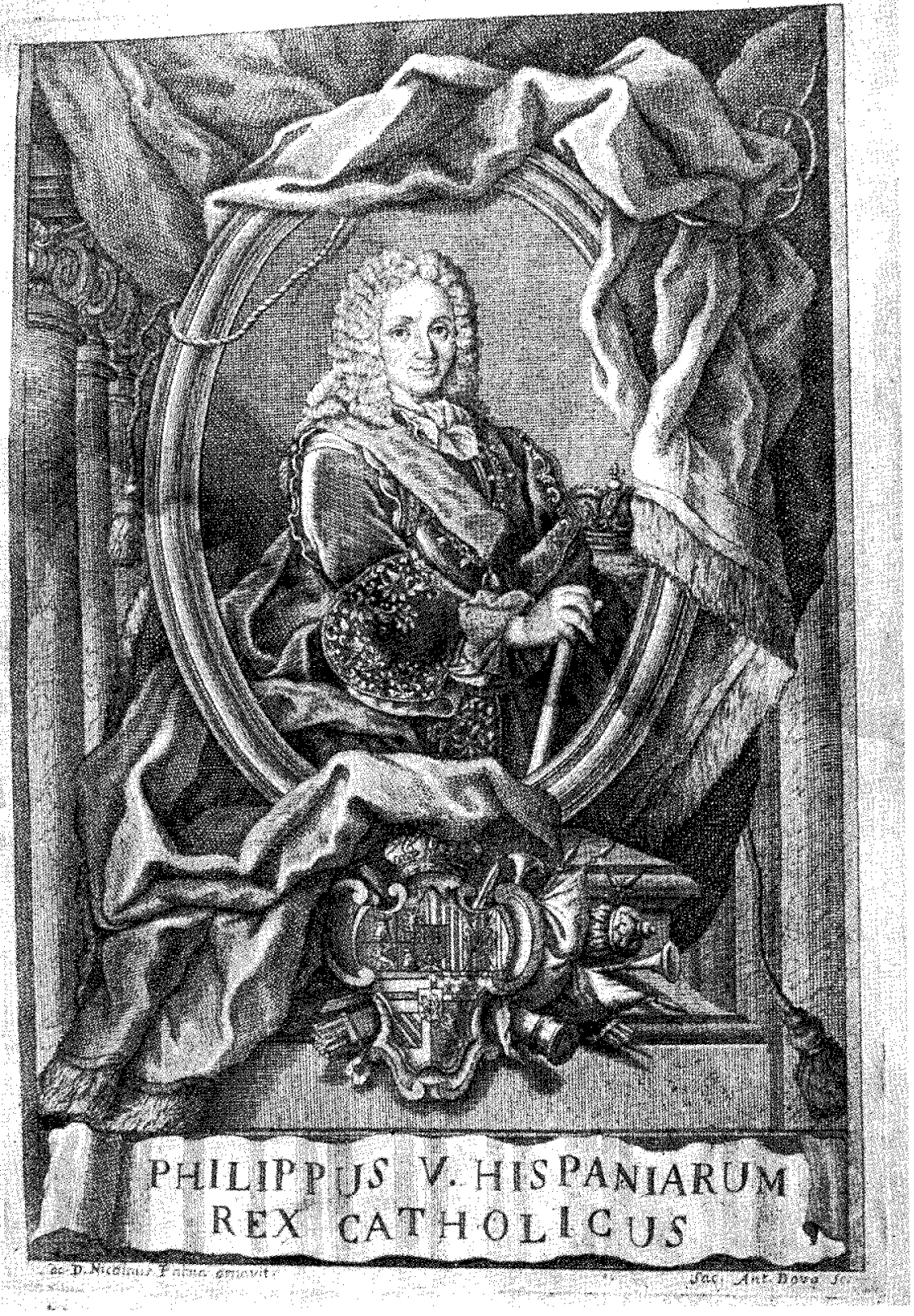

Fig. 2. Anteporta di Esequie Reali per la morte dell'Augusto Re Cattolico Filippo Quinto..., Palermo 1747; ritratto di Filippo $V$. 
pittore. Tale scelta appare in qualche modo giustificata dai rapporti esistenti fra il Fumagalli e la curia arcivescovile, avendo questi realizzato, poco dopo il suo arrivo a Palermo, probabilmente intorno al 1735, «...gli Ornati dagli effetti illusionistici che incorniciano la decorazione di Guglielmo Borremans nel Palazzo arcivescovile di Palermo" ${ }^{26}$. D'altronde il Fumagalli svolse anche l'attività di scenografo e, come pittore, ebbe stretti legami con il mondo architettonico. Sappiamo inoltre che fu coinvolto, anche successivamente, nella progettazione di apparati effimeri, come nel caso delle celebrazioni in onore di S. Giacomo a Caltagirone, nel $1749{ }^{27}$. Che gli apparati del funerale del 1746 non sono opera di un architetto risulta poi evidente, osservando l'incisione del Bova ritraente il catafalco, nella scarsa qualità compositiva dello stesso. I suoi pregi sono più scultorei e pittorici che non architettonici, mancando in esso soluzioni spaziali di rilievo. Interessante è, comunque, la notevole semplificazione operata, soprattutto da un punto di vista ornamentale, rispetto ad antecedenti esemplari pienamente barocchi, spia del graduale mutamento del gusto.

La relazione relativa al funerale del 1746 termina con una sommaria descrizione della funzione a partire dalla «...messa solenne celebrata da Monsignor Ciantro Alfonso Fernandez de Medrano prima dignità di questa Cattedrale, e giudice della monarchia,... ${ }^{28}$, seguita da una breve orazione in lode del defunto re recitata da «...Monsignor Francesco Testa Canonico della medesima chiesa, e Inquisitor fiscale della generale, e suprema inquisizione di Sicilia» ${ }^{29}$.

Se, in generale, tale celebrazione funebre comportò un notevole impegno economico e organizzativo per ottenere uno "spettacolo» che agli occhi degli osservatori risultasse degno del funerale di un re, ben altra cosa fu, comunque, la seconda di tali celebrazioni, organizzata nello stesso edificio. A partire dalla già segnalata data del 17 settembre del $1746, \mathrm{i}$ preparativi si protrassero per circa quattro mesi, ammontando la spesa finale alla notevole círa di seimila scudi, circa ${ }^{30}$; re Carlo aveva dato disposizione che «...dal Reale suo Erario...tutto si ricavasse, quanto era necessario a mettere distintamente in comparsa la Reale Magnificenza, e decretò che suntuosi fussero i Funerali a misura, e tenore di quello stesso

26 M. GutTILLA, Fumagalli..., cit., pag. 213.

27 Ibidem

28 Relazione breve delle solenni esequie di Filippo $V . . .$, cit., pag. 4.

29 Ibidem; anche l'orazione del Testa è stata pubblicata, separatamente dalla relazione relativa al funerale: F. TESTA, Orazione recitata da Monsignor Francesco Testa ne' funerali celebrati dal reverendissimo capitolo e insigne clero della medesima nella morte di Filippo $V$ re delle Spagne, Palermo 1746.

30 F.M. Emanuele e Gaetani marchese di Villabianca, Diario..., cit., pag. 66 
dovere, col quale un contraccambio di Fedeltà, e di vassallagio avea distinto taluno de' passati Monarchi nella Sicilia» ${ }^{31}$. E davvero sontuosi furono i funerali in oggetto (come testimoniano la relazione prodotta per l'occasione e le due incisioni inserite nella stessa ${ }^{32}$ ) la cui celebrazione ebbe inizio alle 22 del 15 gennaio del $1747^{33}$.

La realizzazione dell'apparato effimero e del catafalco predisposti all'interno della cattedrale era stata, in questo caso, affidata a un architetto, Nicolò Palma, come apprendiamo fin dalle prime pagine della relazione di Padre Tamburini ${ }^{34}$. La scelta non sorprende, essendo il Palma, in quella data, Architetto del Senato e Ingegnere Regio, carica, quest'ultima, che probabilmente gli valse l'assegnazione dell'incarico e con la quale, infatti, firma i documenti per l'opera in questione e viene menzionato nelle iscrizioni, a margine delle due incisioni, inserite nel volume della relazione ${ }^{35}$. La prima delle due incisioni ${ }^{36}$ che si incontra nel volume ritrae l'apparato con il quale il Palma andò a ricoprire i sostegni e la sovrastante struttura muraria della navata principale, conferendo alla stessa una nuova foggia. La soluzione architettonica ideata andò, infatti, a occultare del tutto i retrostanti sostegni tetrastili e gli archi a sesto acuto che ancora costituivano la struttura portante all'interno della cattedrale. Al loro posto l'apparato prevedeva l'inserimento di archi a tutto sesto incassati fra robusti pilastri, caratterizzati dall'inserimento

31 Esequie Reali per la morte dell'Augusto Re Cattolico Filippo V..., cit., pag. 39.

32 Si veda la nota precedente; non si tratta in questo caso di un piccolo fascicolo, ma di un consistente volume, accuratamente rilegato, ricco di interessanti particolari e utili annotazioni e descrizioni.

33 Ibidem.

34 IVi, pagg. 49-52; "Appropriato a sostenere il peso della Pompa Lugubre pensò il menzionato Sig. D. Giovanni Maria Ramondetta, che fosse il Rev. Sac. D. Niccolo Palma, che nell'Architettura in ogni qualunque incontro ha dato sempre il miglior conto, ed il più plausibile di se medesimo, secondo le idee, ed il fasto di questa ragguardevole sua Patria, dalla quale fu scelto a porre in maestosa comparsa il carattere luminoso quando si dovettero....prestare gli atti estremi...alla Memoria del Cattolico Re delle Spagne Filippo Quinto Borbone... "; in nota si dice, inoltre, dello stesso Nicolò Palma che, in qualità di Architetto del Senato, fu l'autore degli apparati effimeri per festeggiare l'incoronazione di Carlo III, facendo esplicito riferimento agli apparati per if Palazzo Pretorio e per "...il vago Fonte che di sotto è collocato", pags. 51-55; sempre in nota si legge, inoltre: "Egli è che Ingegnere Reale nel suo impiego è destinato al servigio del Tribunale Supremo della Santissima Inquisizione, e nell'illustriss. ed Eccellentiss. Senato di Palermo eziandio egli è che si adopera, e si affatica alla idea, ed alla esecuzione di quei disegni, che della Insigne Palermitana Grandezza sono l'attestato più veritiero e plausibile...», pagg. 53 e 54.

35 Una leggera discrepanza si riscontra fra documenti e iscrizioni inserite nelle incisioni; nei primi si legge «D. Nicolò Palma, Ingegnere della Regia Corte» (cfr. M.C. RugGiefi Tricol, // «funeral.. cit., Palermo 1993, pags. 43-51), nelle seconde, "Sac. D. Nicolò Palma Ing.ro del Real Patrimonio" (ctr. Esequie Reali per la morte dell'Augusto Re Cattolico Filippo V... cit., Palermo 1747).

36 L'incisione è di Bernardino Bongiovanni, su disegno di Nicolò Palma, secondo quanto si legge nell'iscrizione a margine della stessa: «Sac. D. Nicolò Palma Ing.ro del Real Patrimonio e della Città inventor; Bernardino Bongiovanni Sculp.»; ivi, tav. I. 


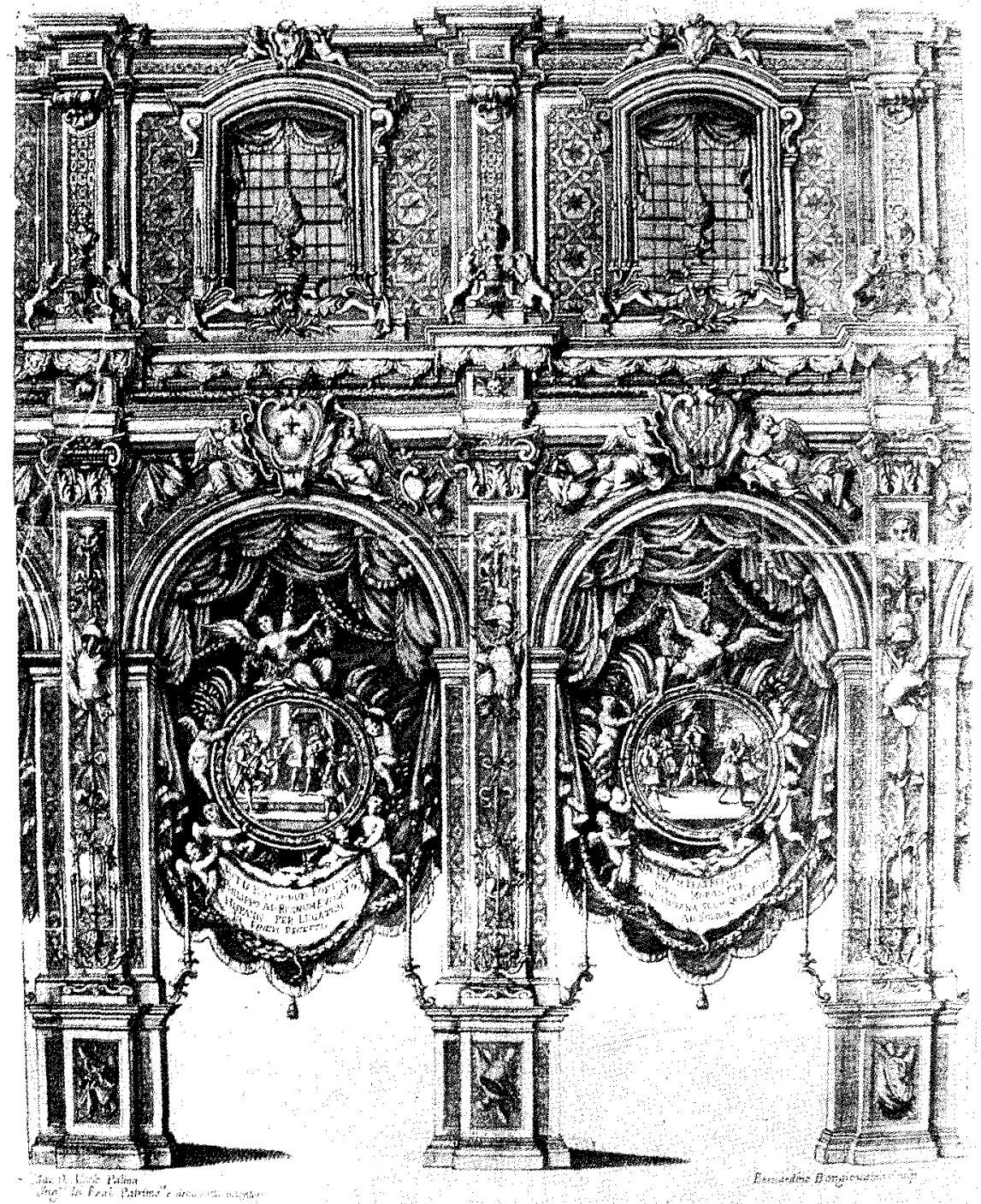

Fig. 3. Apparato per la navata centrale della cattedrale di Palermo, realizzato in occasione delle esequie di Filippo V, celebrate nel 1747; disegno di Nicolò Palma, incisione di Bernardino Bongiovanni (da Esequie Reali..., Palermo 1747). 
di alte paraste composite, che giungevano fino al cornicione, al di sopra del quale principiava il secondo ordine, caratterizzato da una scansione rispondente a quella dell'ordine inferiore. Dalla relazione relativa al funerale apprendiamo che l'architetto aveva adottato una soluzione similare nell'apparato realizzato per la medesima cattedrale in occasione della festa di Santa Rosalia del 1746, di poco antecedente al funerale stesso. II passo in questione per l'esattezza dice: «...poco prima per opera dello esperto mentovato Ingegniero Sacerdote Palma si era segnalata nella Festività della Vergine Concittadina S. Rosalia, imperciocchè cambiando interamente l'aspetto della basilica avea ridotte le basse Colonne, che giungono unicamente a servire di sostentamento alle volte degli Archi proporzionatamente pur bassi nella grandiosa figura di maestosi Pilastri foggiati all'Ordine Corintio, che dal piano sollevandosi per fino ai Capitelli, ed alle volte degli Archi, servivano d'appoggio all'Architrave, e Cornice, che regola il compimento del prim'ordine, oltre del quale il second'ordine delle Finestre ornato di sopra variamente storiato ad emblemi, e figure rappresentava in Teatro luminoso di trionfo, e di allegrezza le virtù, e i tratti della vita prodigiosa, ed austera della Santa Concittadina Romita, per la quale nel vano... de' Pilastri, e nel fondo ricoperto di Specchi apparivano pressoché raddoppiati i lumi distribuiti nella Chiesa ${ }^{37}$ ». La narrazione continua poi con la descrizione dell'apparato predisposto invece, poco dopo, per il funerale di Filippo $\mathrm{V}$, evidenziando le lievi differenze riscontrabili fra le due opere effimere: «della quale fu di bisogno per la Reale celebrazione dei Funerali mutare la scena, e l'ordine stesso dell'Architettura sebbene poco dissomigliante dall'Ordine Corintio, tale più però non si ritenne, ma venne a trasformarsi nell'Ordine Composito, ed i Pilastri architettati nel prim'ordine della Chiesa accrebbero la magnificenza al second'Ordine per guisa che dal piano sino al tetto ingegnosamente tutto fusse concertato con tale artifizio, che spirasse squallidezza, orrore, e lutto, tolto per maggiore serietà della funzione ogni lume, che le aperture delle finestre potevano dare alla Chiesa ${ }^{38}$, volendo, che tutto con-

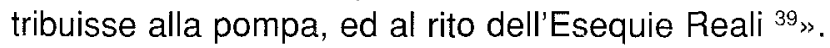

37 IVi, pagg. 86-89.

36 L'oscuramento delle finestre, che non consentiva l'ingresso alla luce naturale proveniente dall'esterno era, però, compensato dalla luce prodotta dalla gran quantità di ceri distribuiti in tutta la chiesa, stando a quanto si legge nella relazione di Padre Tamburini: «...dalla soffitta però stavano sospese le lumiere in numero considerevole, e l'abbondanza dei lumi saliva al novero per fino di seimila, contando quei, che giù pendeano dal tetto della Chiesa, e quei che si erano distribuiti nel Tumolo, dovendosi aggiugnere in oltre ad ogni Pilastro dei 18 Archi un Fanale illuminato da sei fiaccolotti..." (Esequie Reali per la morte dell'Augusto Re Cattolico Filippo V..., cit., pag. 233); in proposito si veda pure quanto riportato in: M.C. RuGGIERI TRICOLI, // «funeral..., cit., pagg. 26 e 27.

39 Esequie Reali per la morte dell'Augusto Re Cattolico Filippo V..., cit., pag. 233. 
Dell'apparato progettato dal Palma per la festa di S. Rosalia non si conserva alcuna riproduzione grafica, ma dalla descrizione sopra inserita risulta evidente che lo schema compositivo adottato era identico a quello che sarà dallo stesso architetto riproposto nei mesi successivi.

Dal confronto con le incisioni ritraenti antecedenti apparati effimeri per la cattedrale, opera dello stesso Nicolò Palma e di altri architetti, emerge in modo evidente il carattere innovativo di tale schema compositivo e, probabilmente, anche una volontà di rinnovamento della veste architettonica con cui si presentava la cattedrale. In generale si nota una razionalizzazione dell'immagine architettonica, con una semplificazione della struttura e, in particolar modo, dei sostegni, dai quali scompaiono del tutto le colonne e che per la prima volta vengono innalzati fino a sostenere il cornicione di coronamento del primo ordine, andando gli archi a tutto sesto a incassarsi, in modo analogo a quanto attuato nella trasformazione tardosettecentesca della cattedrale, basata sul progetto di Ferdinando Fuga. Indubbio accostamento alla corrente classicista che andava prendendo piede in varie parti d'Italia, tale cambiamento di rotta da parte del Palma, evidente se facciamo un paragone fra l'apparato in questione e quelli dallo stesso ideati, in precedenza, per l'acclamazione di re Carlo (nel 1735) ${ }^{40}$, o ancora per le nozze dello stesso sovrano (nel 1739) ${ }^{41}$, dipese, probabilmente, da un generale mutamento del gusto.

Per quel che concerne, in modo specifico, la cattedrale, siamo in un momento di pochi anni successivo alla visita regia di De Ciocchis (1741) ${ }^{42}$ e di pochi anni anteriore alla perizia di Giovan Battista Vaccarini ${ }^{43}$. Si tratta per-

40 L'apparato in questione, progettato da Nicolò Palma, è stato riprodotto in un incisione di Antonino Bova inserita all'interno di: P. LA PLACA, La Reggia in Trionfo per l'acclamazione, e coronazione della Sacra Real Maestà di Carlo, Palermo 1736.

${ }^{41}$ Anche di questo apparato, frutto dell'ingegno del medesimo architetto, è stata realizzata un'incisione dallo stesso Antorino Bova inserita in: P. La Placa, Relazione delle Pompe Festive seguite in Palermo Capital della Sicilia nella celebrità delle regie nozze di Carlo di Borbone Re di Sicilia, e di Napoli, con Maria Amalia Principessa di Polonia, e di Sassonia, Palermo 1739.

42 Si tratta di un regio visitatcre apostolico che nell'agosto del 1741 redasse una relazione sullo stato delle fabbriche della cattedrale di Palermo, richiedendo «... riparazioni al tetto, alle murature esterne, ma soprattutto opere plastiche, di ornamento, di decorazione e di imbiancamento dei portici ecc., a conferma che in quegli anni si riteneva di poter svolgere opere di manutenzione, ma soprattutto di abbellimento della chiesa esistente" (S. BOSCARINO, La "restaurazione" della Cattedrale nel Settecento, in La Cattedrale di Palermo. Studi per lottavo centenario della fondazione, a cura di L. URBANI, Palermo 1993, pag. 95). La relazione in questione è stata pubblicata in: J. A. De Ciocchis, Sacrae Regiae Visitationis per Siciliam... acta decretaque omnia, Palermo 1836.

43 La perizia eseguita dal Vaccarini, su commissione dell'arcivescovo Melendez, risale al marzo del 1752. Non essendosi conservato alcuno scritto, né alcun grafico relativo alla stessa risulta impossibile indicare con certezza quale fosse il suo contenuto. In proposito scrive Maria Giuffrè che la perizia del Vaccarini potrebbe essere stata una semplice relazione preventiva agli 


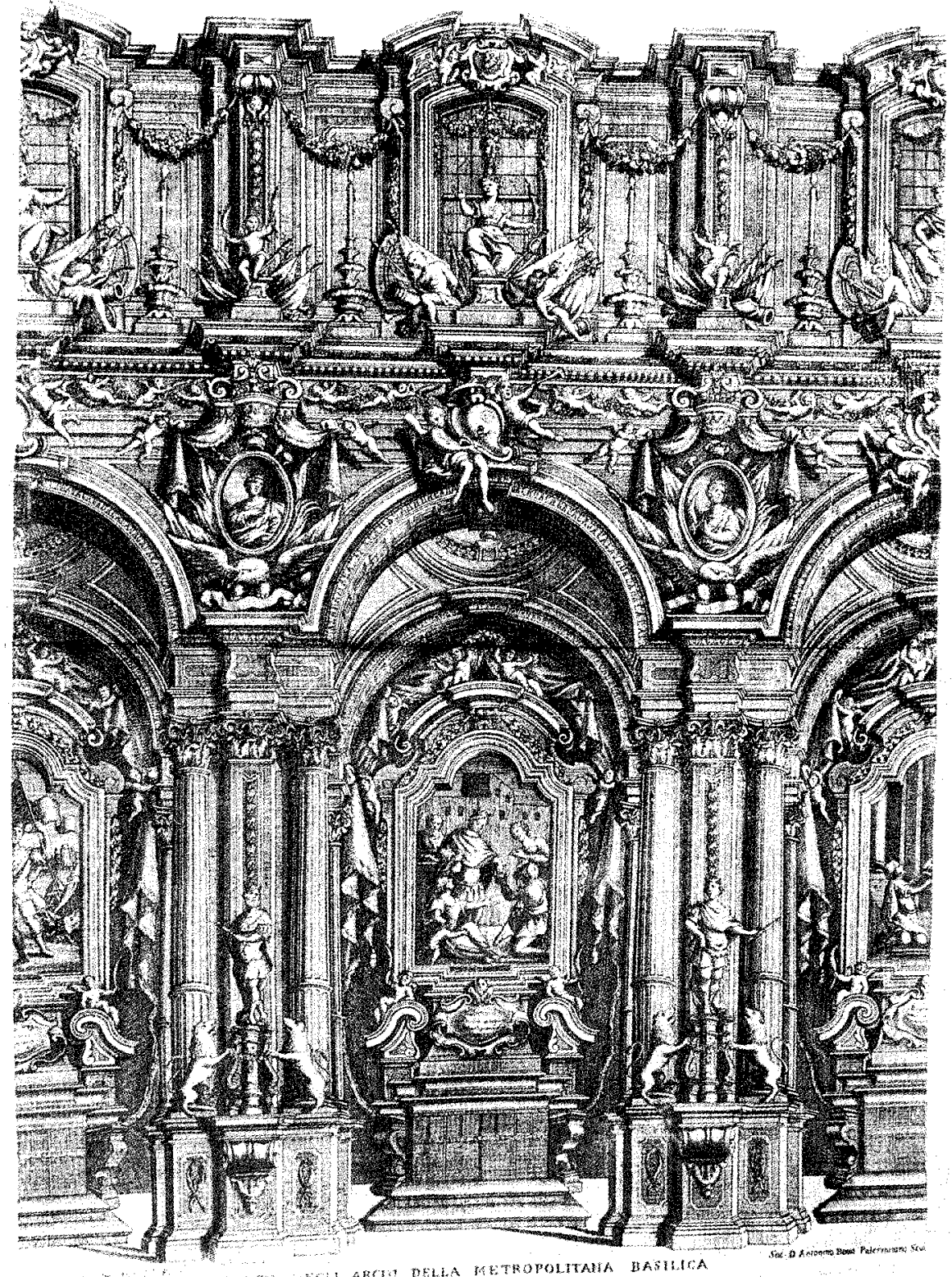

Fig. 4. Apparato per la navata centrale della cattedrale di Palermo, realizzato in occasione dell'acclamazione di re Carlo (1735); disegno di Nicolò Palma, incisione di Antonino Bova (da P. La Placa, La Reggia in Trionfo..., Palermo 1736). 
tanto di un periodo antecedente le grandi polemiche che portarono alla stesura del progetto di Fuga per l'ammodernamento della spazialità interna della chiesa. E' tuttavia probabile che si registrassero già le prime lamentele per il disordine e l'eccessiva eterogeneità della stessa. Una prima spia di tale diffuso sentimento potrebbe essere individuata in due righe del passo, tratto dalla relazione per i funerali di Filippo V, riportato in precedenza, in cui, a proposito dell'apparato progettato dal Palma per la festa di S. Rosalia, si dice: «...della basilica avea ridotte le basse Colonne, che giungono unicamente a servire di sostentamento alle volte degli Archi proporzionatamente pur bassi, nella grandiosa figura di maestosi Pilastri... ${ }^{44}$. E' possibile, quindi, che al di là della retorica che caratterizza questo tipo di letteratura, l'esaltazione dell'apparato effimero proposto dal Palma derivi da una diffusa convinzione che l'interno della cattedrale e i suoi sostegni, in particolar modo, andassero modificati.

La celebrazione dei funerali di Filippo $V$ nel 1747 e l'attribuzione dell'incarico per la progettazione degli apparati effimeri potrebbero pertanto essere state per Nicolò Palma l'occasione ideale per affrontare il problema della spazialità interna della cattedrale e di una sua possibile riconfigurazione. Il disegno di tale apparato, di conseguenza, sarebbe stato dall'architetto concepito non come semplice esercitazione, in cui dare sfogo alla propria fantasia ${ }^{45}$, ma come vera e propria idea progettuale da proporre a un pubblico straordinariamente vasto. Dai documenti fino ad cggi studiati non risulta alcun coinvolgimento del Palma nelle annose vicende che interessarono la cattedrale palermitana a partire dagli anni sessanta del Settecento, tranne che per la sua presenza, nel 1772, all'interno di una commissione di periti che, nelle more dell'approvazione del progetto del Fuga, compì una visita di controllo all'edificio della cattedrale «...i cui esiti vengono esplicitati due anni dopo -il 2 febbraio 1774-» 46 in una relazione firmata dallo stesso Nicolò Palma ${ }^{47}$. Risulta pertanto

interventi di manutenzione, forse «straordinaria», da effettuare sulla fabbrica della cattedrale, anche se non esclude la possibilità che l'architetto abbia prodotto in tale occasione un vero e proprio progetto di trasformazione deila stessa (M. Guffrè, Il cantiere della Cattedrale di Palermo da Ferdinando Fuga a Emanuele Palazzotto, in La Cattedrale..., cit., pagg. 257-260).

44 Si veda la nota 35.

45 Il carattere sobrio e, soprattutto, tettonico della struttura progettata potrebbe esserne la riprova. Si tratta, cioè, a differenza degli apparati proposti dallo stesso architetto in precedenza (si vedano le note 40 e 41), decisamente atettonici, di uno schema compositivo strutturalmente corretto e attuabile in un edificio reale.

46 M. Giuffrè, I/ cantiere della Cattedrale... cit., in La Cattedrale..., cit., pag. 257.

47 Ibidem; in questo caso Palma si firmò Architetto del Senato; lo stesso Nicolò Palma firmò, inoltre, la relazione di un'antecedente visita dallo stesso condotta fra 1753 e '54, in cui l'interesse è però puntato sulla «balaustrata del piano della Cattedrale» (S. BoscARINO, La «restaurazione»..., cit. , in La Cattedrale..., cit., pag. 95). 


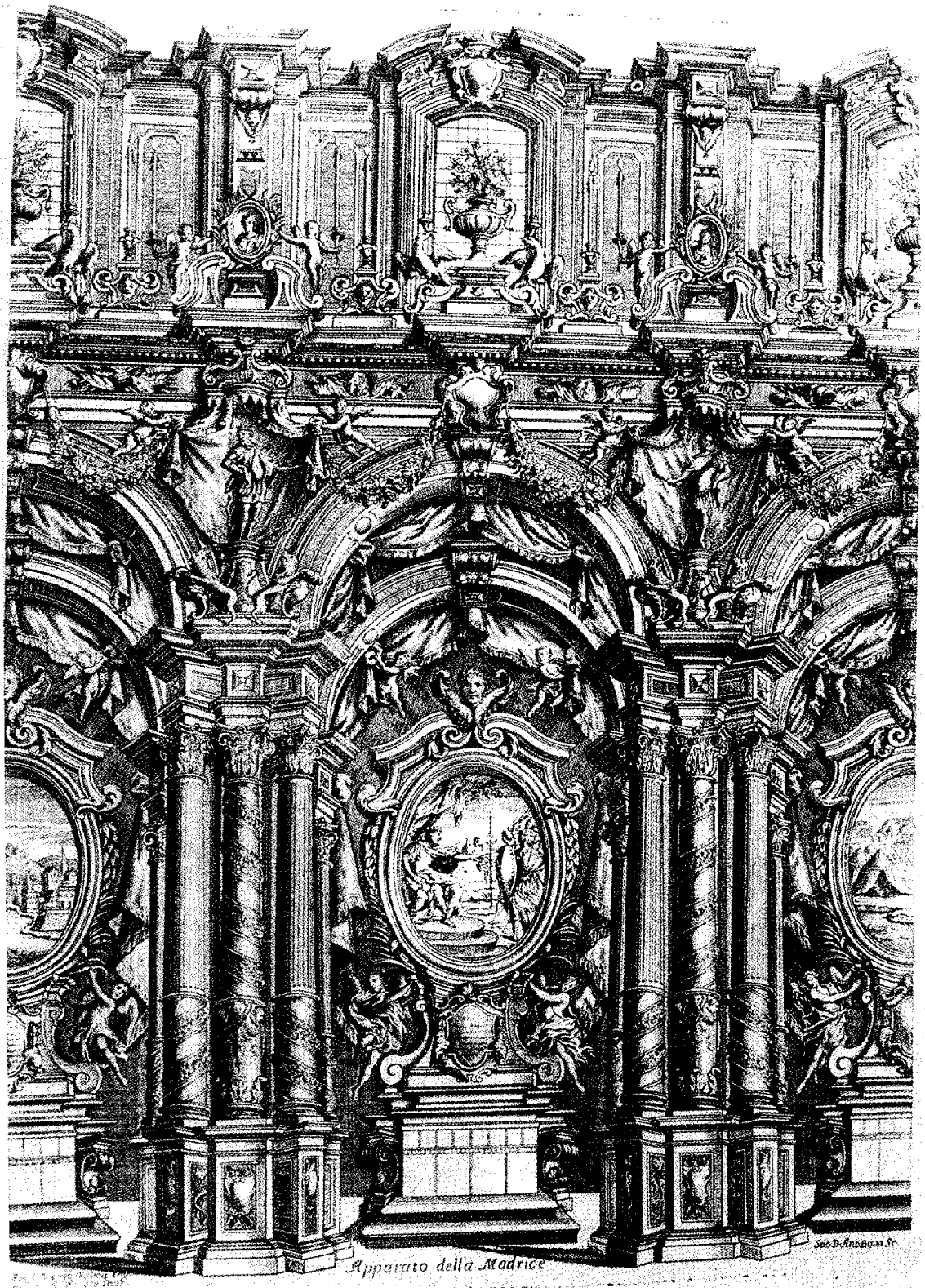

Fig. 5. Apparato per la navata centrale della cattedrale di Palermo, realizzato in occasione delle nozze di re Carlo (1739); disegno di Nicolò Palma, incisione di Antonino Bova (da P. La Placa, Relazione delle Pompe Festive ..., Palermo 1739). 
difficile comprovare l'esattezza dell'ipotesi da noi avanzata, non avendo alcun riscontro della posizione presa dal Palma nel dibattito creatosi intorno al progetto del Fuga, fra sostenitori e oppositori dello stesso, per quanto l'indubbia affinità fra quest'ultimo e l'apparato per i funerali di Filippo $\mathrm{V}$ potrebbe di per sé costituire una risposta. Si tratti o meno di una velata proposta progettuale per la trasformazione dell'interno della cattedrale palermitana, l'apparato effimero in esame è, comunque, di grande interesse. Esso costituisce infatti un significativo saggio dell'evoluzione del linguaggio architettonico di Nicolò Palma, personaggio di primo piano nel panorama architettonico palermitano fra la prima e la seconda metà del Settecento ma ancora da decifrare nelle sue molteplici valenze culturali.

Tornando alla descrizione dell'apparato effimero, ulteriori indicazioni, relative alla finitura delle superfici dell'apparato stesso e ai motivi decorativi più ricorrenti, sono contenute nella relazione del funerale: «...quello che nell'architettura formava tutto il massiccio, e quello ch'era il fondo era tutto di argento, e quanto serviva all'intorno per ornamento, e contorno, quanto vi era d'intaglio, e cornice era pomposamante messo ad oro, il fondo poi risaltava più di qualunque altra cosa, perché di Mosaico scuro proporzionato nel tempo stesso a dimostrare lutto, e scoruccio, e magnificenza, e maestà» ${ }^{48}$. Sulla decorazione poi, in particolare, si legge: «...si disegnarono rilevati sul bronzo Trofei militari, dei quali fu giusto pensiero dell'Architetto mettere in comparsa una quantità folta, e numerosa; ne attaccò perciò moltissimi ai fondi dei piedistalli, ne appese ai pilastri, ne fregiò tutte le parti...a divisare maestosamente il bellicoso Genio guerriero dell'estinto monarca» ${ }^{49}$. La relazione prosegue quindi con la descrizione del motivo decorativo introdotto in corrispondenza della chiave di ciascuno degli archi, incassati fra i sostegni della navata principale: una «...Targa dorata sostenuta con brio da due Geni, effigiati in argento, nel mezzo della quale era dipinto lo stemma particolare di cadauno dei Regni soggetti... ${ }^{50}$. Diverse pagine, infine, sono dedicate al racconto delle immagini dipinte, raffiguranti i momenti più significativi della vita del defunto re, inserite nei medaglioni, contornati da ricchi elementi ornamentali ${ }^{51}$, sospesi al di 93 e 94 .

48 Esequie Reali per la morte dell'Augusto Re Cattolico Filippo V... cit., Palermo 1747, pagg.

$49 \quad$ Ivi, pagg. 94 e 95.

$50 \mathrm{IVi}$, pagg. 98.

51 IVi pagg. 98-102: «...un gruppo di Genietti volanti scopriva un medaglione circolare di basso rilievo col fondo di bronzo, che si era fatto incorniciare d'oro, e pendea giù dall'arco per mezzo di una lunga catena, sostentato altresì da varj Geni figurati in argento, dei quali altri ricolme avean le mani di palme, altri fiori d'oro andavan congegnando all'intorno, e molti sostenevan per fine una larga pelle di Leone messa al di fuori riccamente ad oro, e col fondo al di dentro tirato sopra l'argento, ove si dovettero segnare a Spettatori le intraprese, per le quali nel suo vivere si distinse il Magnanimo Re...". 


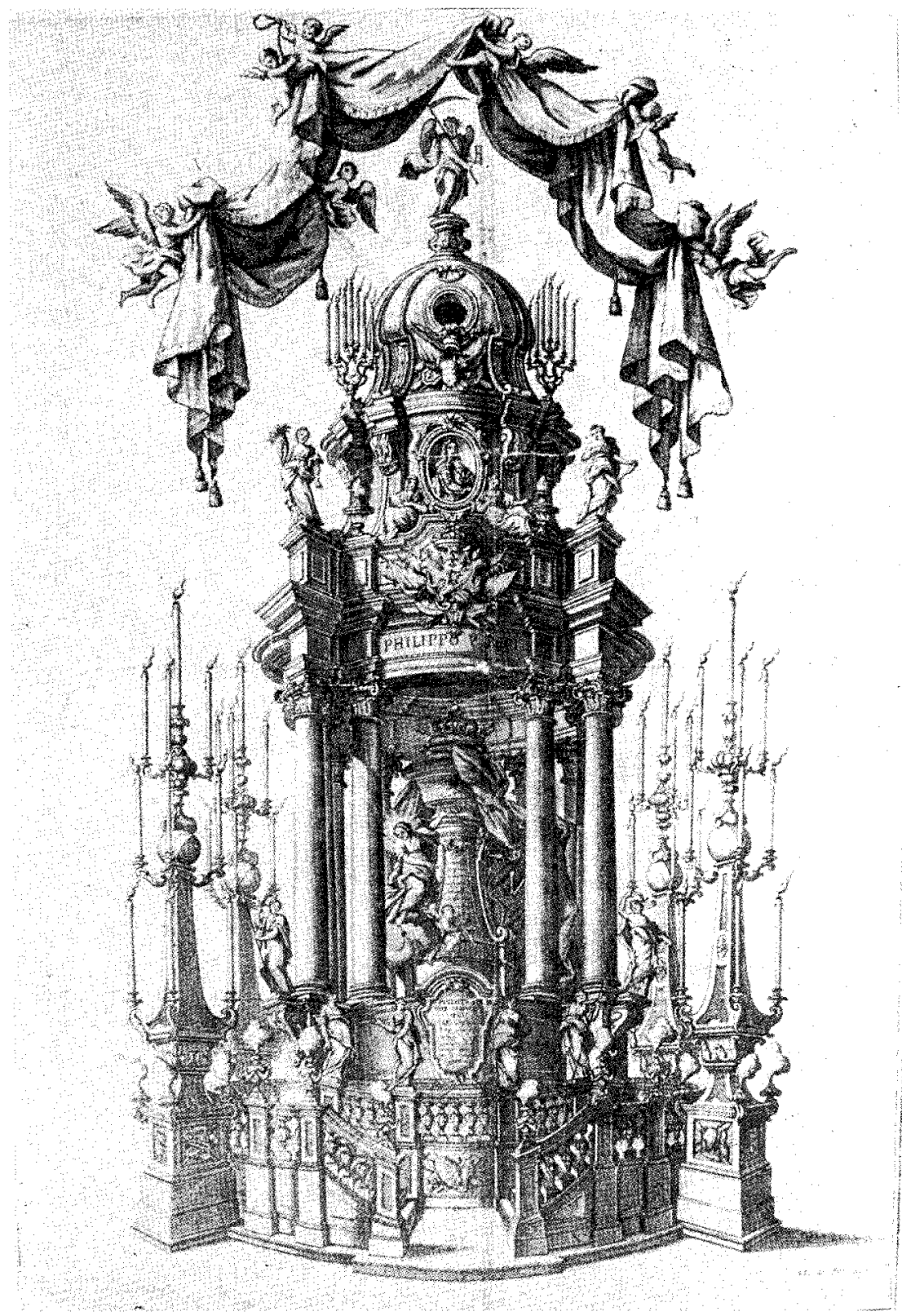

Fig. 6. Catafalco eretto allinterno della cattedrale di Palermo in occasione delle esequie di Filippo V, celebrate nel 1747; disegno di Nicolò Palma, incisione di Antonino Bova (da Esequie Reali..., Palermo 1747). 
sotto degli stessi archi, nove per lato, fino alle Cantorie, fra drappi neri, guarniti di frange e festoni d'oro e d'argento.

Le immagini pittoriche, distribuite negli interassi dei sostegni della navata centrale, segnavano le successive tappe di un percorso, all'interno della cattedrale, architettato dal Palma, avente come fulcro e momento di somma maestosità il grandioso catafalco, ubicato «innanzi il coro in mezzo alla nave» ${ }^{52}$. Nella relazione di Padre Tamburini in proposito si legge: «...la magnificenza però, e la ricchezza risaltavano maggiormente verso il finimento della stessa nave di mezzo: ove si costrusse verso le Cantorie un grandioso Tumulo dipinto, e figurato con adornamenti sfarzosi, che sopra il fondo di mosaico spargeano finimenti d'oro, d'argento e di bronzi a vicenda mirabilmente intrecciati; si sollevava la eccelsa macchina fino all'altezza di palmi 80, e prima sorgeva in essa da terra alquanti palmi un piano di pianta circolare, alla quale salivasi pure per quattro ordini di scalinate circolari ancor esse, ed all'intorno chiudevasi esso piano con varie balaustrate, vi si aggiunsero profumiere per ornamento, e di sedici statue di statura ottopalmare, donde servia cadauna a rappresentare le insigni Virtù dell'estinto Monarca Filippo Quinto dipinte...e scolpite ad uso di bianco marmo, ordinate al risalto della funebre mole architettata col disegno del mentovato Sarcedote D. Niccolò Palma» ${ }^{53}$. Segue, poi, nelle successive pagine della relazione, un'accuratissima descrizione dei diversi ordini ed elementi architettonici e decorativi che caratterizzavano la grandiosa «macchina» funebre, a partire dal piano che sovrastava la zona basamentale, descritta nel passo sopra riportato ${ }^{54}$. Lo stesso catafalco è,

52 F.M. Emanuele e Gaetani marchese di Villabianca, Le feste reali.., cit., pagg. 165.

53 IVi, pagg. 200 e 201

54 «...seguitando poi l'ordine dell'incominciata Architettura nel mezzo dell'avvisato Piano circolare era situato un altro rialzamento, che serviva di basamento al gran piedistallo dell'Urna Reale, la quale medesimamente ufficio di piedistallo facea a dodici ben ordinate colonne, sostenenti una volta Reale di una Tribuna, che venia a formarsi, entro la quale la stessa Urna Reale era maestosamente racchiusa. II modello dell'Architettura avea formate queste colonne d'ordine composito, e scanellate, con tale regola, e disposizione, che conservassero fra di loro a due a due la pianta primiera circolare; delle stesse colonne però uscendo fuori di quella quattro, e risaltando fra le altre la rendeano graziosamente centinata, ed a piè delle accennate quattro colonne, e da loro ben architettati piedistalli sporgeano pure allo infuori quattro contornate mensole, sopra le quali altrettante Statue posavano, dirette a significare la vita condotta fra gli atti di perfetta virtù...", in Esequie Reali per la morte dell'Augusto Re Cattolico Filippo V..., cit., pagg. 212 e 213; proseguendo, ancora in merito al catafalco, si legge: «...Ritornando a favellare di quello, che dava a vedere l'Architettura, torniamo pure a fissarsi nella stessa Pianta circolare, e nobilmente centinata, ed in essa sulle dodici descritte colonne posavano con ordine l'architrave, fregio, e cornice ed in lettere cubitali riccamente messe ad oro era scritto nel fregio stesso così: PHILIPPO V. BORBONIO. HISPANIARUM. INDIARUMQU. REG. CATHOLICO», ivi, pag. 215; e ancora: «...innalzandosi dall'Architetto un altro ordinetto sopra del primo si collocarono sopra dei piedistalli quattro statue differentemente abbigliate, esprimenti le quattro Parti del Mondo, e posavano queste su quella 
inoltre, ritratto nella seconda incisione inserita nella relazione pubblicata in occasione del funerale ${ }^{55}$. Dalla lettura della relazione e dall'osservazione dell'incisione è possibile dedurre tutte le informazioni necessarie per ricostruire con precisione il progetto di Nicolò Palma per il catafalco di Filippo V.

Si tratta anche in questo caso di un progetto estremamente interessante per le indicazioni che fornisce in merito all'evoluzione del linguaggio architettonico del Palma «verso quel nitore geometrico, quei tagli netti, quella severità d'ornati...via via di gusto sempre più classico» 56 . II catafalco in questione, caratterizzato da una notevole eleganza e da un certo verticalismo, segna, inoltre, una frattura rispetto alle analoghe macchine progettate in precedenza a Palermo e mostra interessanti affinità con quello progettato dal Sacchetti per il funerale in suffragio del medesimo monarca Filippo V, celebrato nel 1746 nel convento della chiesa della Encarnaciòn di Madrid ${ }^{57}$. E', in particolare, la presenza di una sorta di tempietto a pianta circolare, inusuale per la realizzazione di macchine funebri anche in ambito spagnolo ${ }^{58}$, ubicato al di sopra di un più o meno articolato zoccolo basamentale ${ }^{59}$, ad avvicinare i due progetti e a suggerire una comune

parte delle colonne, che risaltava all'infuori, e che servia eziandio a sostenere otto mensole, che prendendo tutta la direzione dell'altre otto colonne si alzavano a reggere il cimazio, l'imboccatura della Cupola, che per un adatto traforo avea graziosamente il suo finimento; ...nell'accennato ordinetto nei quattro centri dei principali prospetti si dovette collocare un adornamento, ..., così furono disegnati quattro scudi... ove s'inquadrarono primieramente i Stemmi allusivi all'Armi Reali, e...in Ovati si formarono quattro bassirilievi:...Si chiudea finalmente al di sopra la mole maestosa, e magnifica con una Statua appropriata a significare il Tempo:..., sopra del quale sostenuto da lunghi cordoni era un ordine di cascate di velluto con trine, e frangie», ivi, pagg. 216-221; inoltre :«...; la Tribuna poi, della quale posava nel mezzo l'Urna Reale, tutta si era graziosamente abbeIlita a bassi rilievi....I fondo era tutto d'argento, e d'argento erano pure i contorni dei bassorilievi...", ivi, pag. 222; e infine: «..., non bastò tuttavia quanto si vedea ad appagare il genio del Valente Architetto Sac. Palma, che attorno della stessa Mole dispose quattro Obelischi ripartiti a quattro lati del medesimo Tumulo, dai quali ingegnosamente traforati uscivano odorosi profumi, ed erano pur essi carichi a dismisura di cere,... disegnare su'l fondo degli obelischi medesimi Trofei, ed Imprese militari,... », ivi, pag. 232.

55 Si tratta di un'incisione eseguita da Antonino Bova, su disegno di Nicolò Palma, il cui nome compare, in questo caso, nell'iscrizione inserita a margine della stessa, affiancato dai titoli di Ingegnere del Real Patrimonio e ... della Città.

56 M.C. RugGieri TRICOLI, // «funeral..., cit., pagg. 24 e 25.

57 Si veda in merito: V. Soto CABA, Catafalcos reales del Barroco Español. Un estudio de arquitectura efimera, U.N.E.D., Madrid 1991, pagg. 289-303.

$58 \quad$ Ivi, pag. 292.

59 La macchina progettata dal Sacchetti presenta nella zona basamentale una maggiore complessità compositiva, rispetto a quella di Nicolò Palma. La prima presenta, infatti, nello zoccolo, decisamente più alto rispetto a quello della seconda, un'apertura arcuata che metteva in comunicazione la zona del coro con quella dell'altare maggiore, consentendo la visione di quest'ultimo dalla navata principale; ivi, pag. 291. 


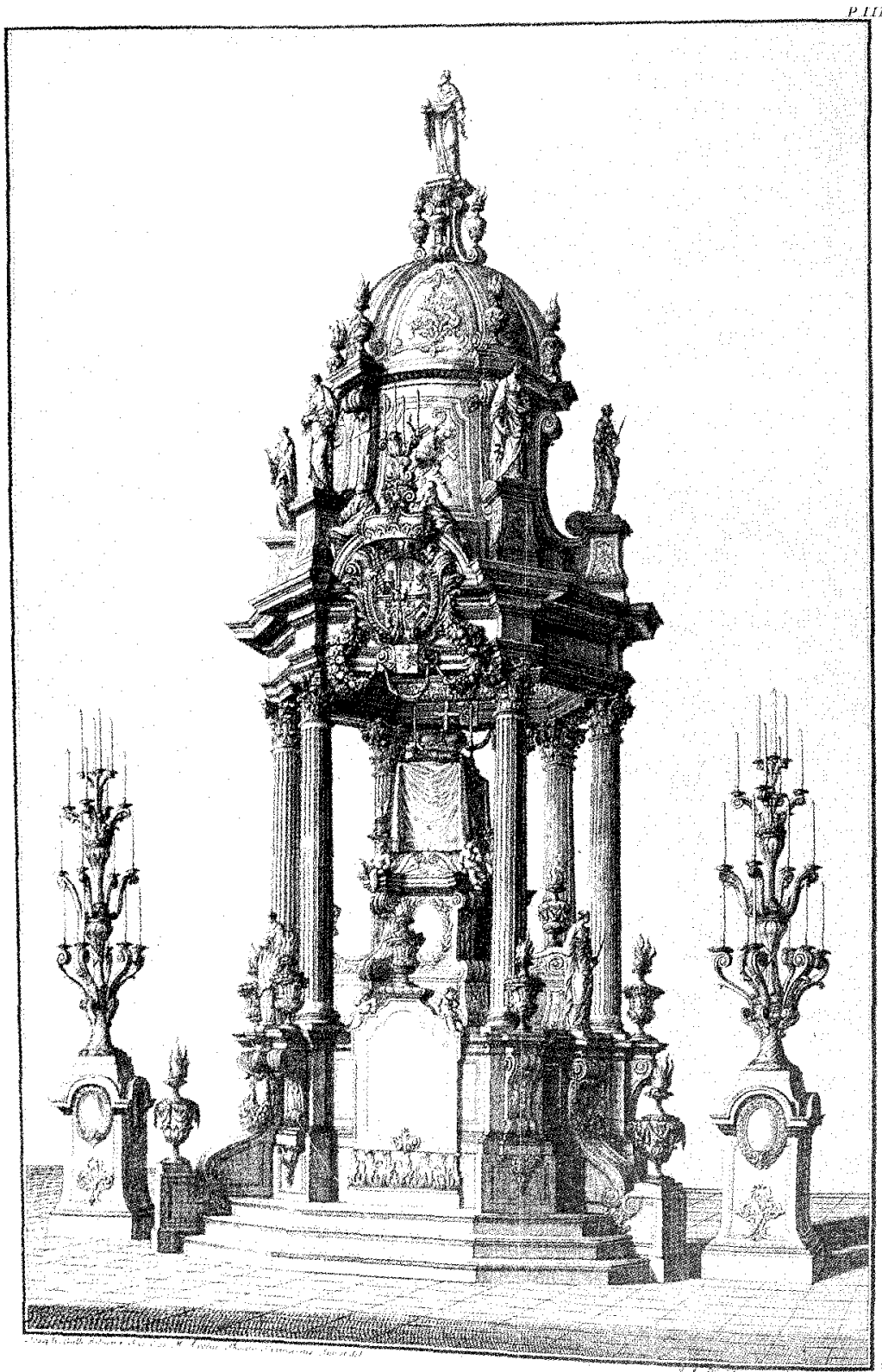

Fig. 7. Progetto per un catafalco; disegno di Giuseppe Galli Bibiena (da Architetture, e prospettive dedicate alla Maestà di Carlo Sesto ..., Augusta 1740). 
matrice per entrambi: i progetti per catafalchi prodotti da Giuseppe Galli Bibiena per la Corte viennese ${ }^{60}$.

Fra i possibili modelli che ispirarono il Palma nell'invenzione del catafalco per Filippo $\mathrm{V}$, va inoltre segnalato il progetto di Filippo Juvarra per l'altare della chiesa di S. Ignazio Martire all'Olivella di Palermo, commissionatogli nel 1715. Quali fossero le caratteristiche di tale progetto è possibile dedurlo da tre schizzi, attualmente custoditi presso la Biblioteca Nazionale Universitaria di Torino, riferibili al progetto in questione ${ }^{61}$. Dello stesso fu realizzato, nel 1722, un modello ligneo, utilizzando gli schizzi sopra menzionati, che poi "probabilmente fu tradotto al vero come testimonia il superstite tabernacolo» ${ }^{62}$. Nicolò Palma avrà certamente preso visione di tale progetto ed è pertanto plausibile che dallo stesso abbia potuto trarre spunto per il catafalco di Filippo $V$, ipotesi avvalorata dalle indubbie affinità compositive ${ }^{63}$.

Dalla relazione redatta in occasione del funerale del 1747, infine, apprendiamo che l'architetto intervenne anche nella zona del coro, nella quale, per consentire alle «Dame» di assistere alla solenne funzione, «...sopra i Stalli medesimi del Capitolo si architettarono due Tribune, alle quali salivano le Dame per due scalinate ben concertate a misura della comodità, e del bisogno da ambedue le parti..." ${ }^{64}$. In definitiva, quindi, per l'allestimento dei regi funerali la cattedrale di Palermo venne, in entrambi i casi presi in esame, interessata nella sua globalità (dalle porte d'accesso ${ }^{65}$ alle navate, dal coro all'altare maggiore ${ }^{66}$ ) da interventi architettonici e decorativi a carattere effimero, necessari sia per adeguare la stessa al fasto che tali celebrazioni presupponevano, che per renderla

60 Un folto numero di disegni scenografici di Giuseppe Galli Bibiena, fra cui diversi raffiguranti catafalchi, erano stati riprodotti in un gruppo di incisioni, cinquanta per l'esattezza, pubblicate nel 1740 (Architetture, e prospettive dedicate alla Maestà di Carlo Sesto Imperador de' Romani da Giuseppe Galli Bibiena, suo Primo Ingegner teatrale, ed Architetto inventore delle medesime, Augusta 1740). E' pertanto probabile che i due architetti conoscessero tali incisioni.

61 Si veda in merito: C. D'Arpa, Un progetto di Filippo Juvarra per un altare a Palermo, in "॥ Disegno di Architettura», n. ${ }^{\circ}$ 18, Milano, novembre 1998, pagg. 47-49.

62 IVi, pag. 47.

63 Ivi, pag. 49; l'autore dell'articolo segnala, inoltre, due realizzazioni palermitane a piccola scala che risentirono probabilmente dell'influsso esercitato dal progetto juvarriano: i cibori collocati sugli altari delle chiese di S. Caterina al Cassero e di S. Chiara.

64 Esequie Reali per la morte dell'Augusto Re Cattolico Filippo V..., cit., pag. 234.

65 Ivi, pagg. 65-78; la descrizione dell'apparato effimero per la navata centrale è preceduta, nella relazione del 1747, dalla descrizione degli ornamenti apposti a tutte le porte d'accesso alla cattedrale, iniziando dalla porta maggiore.

${ }_{66} \quad$ Ivi, pag. 235; «...; l'Altar Maggiore eziandio si era pomposamente accomodato, massimamente nel fondo con panni neri trinati, per tutt'i lati gli facea ingegnosamente contorno una Cortina ricca per tutte le parti di trine, e frangie, e più lumiere pendevano giù dal tetto...” 
rispondente alle necessità pratiche che le modalità di svolgimento delle stesse celebrazioni richiedevano.

A conclusione dei ragionamenti occorre inserire un'ultima considerazione: $i$ testi a stampa prodotti in tali frangenti veicolano talora ambizioni non apertamente dichiarate, ma leggibili fra le righe, che vanno ben oltre if semplice racconto delle «esequie solenni». E' questo probabilmente il caso del voluminoso libro pubblicato nel 1747, il cui intento non dichiarato era forse quello di iniziare a pubblicizzare proposte di intervento nella cattedrale. Una rilettura dei testi, che non si fermi a un livello superficiale, può ancora fornire fertili spunti, suggerendo indizi per l'interpretazione di fenomeni ed eventi che esulano dai ristretti confini dell'effimero. 